Case Report

\title{
Idiopathic Thrombocytopenic Purpura after Mastectomy and Axillary Lymph Node Dissection
}

\author{
Wil L. Santivasi, Meghan M. Routt, and Alicia M. Terando \\ Division of Surgical Oncology, Department of Surgery, The Ohio State University College of Medicine, N924 Doan Hall, \\ 410 W 10th Avenue, Columbus, OH 43210, USA \\ Correspondence should be addressed to Alicia M. Terando; alicia.terando@osumc.edu
}

Received 18 December 2013; Accepted 5 February 2014; Published 6 March 2014

Academic Editors: D. Mantas, A. Nakajo, O. Olsha, and C. Tunon-de-Lara

Copyright (C) 2014 Wil L. Santivasi et al. This is an open access article distributed under the Creative Commons Attribution License, which permits unrestricted use, distribution, and reproduction in any medium, provided the original work is properly cited.

First described in 1916, idiopathic thrombocytopenic purpura (ITP) is an autoimmune disease resulting in the destruction of platelets. Here, we present a case of an 85-year-old patient diagnosed with invasive ductal carcinoma of the breast whose surgical treatment was complicated postoperatively by acute-onset thrombocytopenia with a resultant hematoma at the operative site. Diagnostic Workup revealed no clear etiology for the thrombocytopenia; therefore, a presumptive diagnosis of idiopathic thrombocytopenic purpura was made. Previous literature has associated the development of idiopathic thrombocytopenic purpura with breast cancer. However, to the authors' knowledge, there are no reported cases of ITP presenting immediately following surgical intervention for breast cancer in the absence of other etiologic factors.

\section{Introduction}

Idiopathic thrombocytopenic purpura (ITP) is an autoimmune disease, first described in 1916 [1], in which autoantibody production results in destruction of platelets and thrombocytopenia [2]. Prior reports of ITP in patients diagnosed with breast cancer exist where ITP preceded the diagnosis of breast cancer [3] or occurred during adjuvant therapy $[4,5]$ or metastatic breast cancer was discovered upon therapeutic splenectomy for ITP [6]. Here, we report a case of idiopathic thrombocytopenic purpura which presented after mastectomy for invasive ductal carcinoma.

\section{Case Report}

An 85-year-old Caucasian, postmenopausal female presented to the outpatient clinic with a palpable mass in her left breast. Initial diagnostic mammography identified spiculation and architectural distortion inferior to the $2.1 \mathrm{~cm}$ mass at the 12 oclock position of the left breast. Targeted left breast ultrasound demonstrated two distinct hypoechoic lesions in the 12:00 axis of her left breast, which was classified as BIRADS-5. Ultrasound-guided biopsy revealed invasive ductal carcinoma (ER positive, PR positive, and Her2-Neu negative) in three different areas within her left breast. Left modified radical mastectomy with sentinel lymph node biopsy and axillary lymph node dissection was performed. Three of six sentinel lymph nodes were found to be positive on frozen section (one with macrometastasis and two with micrometastasis), and a full axillary lymph node dissection was performed.

The patient was convalescing satisfactorily until the afternoon of postoperative day one, when the output of her surgical drains became sanguineous and voluminous. A pressure dressing was applied to the chest wall. On postoperative day two, her hemoglobin had dropped to $8.8 \mathrm{~g} / \mathrm{dL}$ from $13 \mathrm{~g} / \mathrm{dL}$ preoperatively, and her platelet count decreased from $132,000 / \mathrm{mm}^{3}$ to $8,000 / \mathrm{mm}^{3}$. A fluid collection was noted along the length of her incision, primarily in the left axilla. She had extensive bruising along the incision and axilla, tracking downward on the abdomen. Despite these findings, the patient remained asymptomatic and hemodynamically stable. A peripheral blood smear demonstrated no cellular fragmentation. A review of the patient's current medications 
revealed no potential cause for a drug-induced thrombocytopenia, and a review of her past medical history revealed no other possible etiologies for thrombocytopenia. Given this, the patient was diagnosed with presumptive idiopathic thrombocytopenic purpura (ITP).

Beginning on postoperative day two, the patient began receiving $40 \mathrm{mg}$ prednisone daily, and she received platelet transfusion. Following these measures, her platelet count increased to $87,000 / \mathrm{mm}^{3}$ and continued to improve thereafter. She was discharged from the hospital on postoperative day four in stable condition with a platelet count of $186,000 / \mathrm{mm}^{3}$. Her systemic steroids were then tapered over the course of four weeks.

\section{Discussion}

ITP is a relatively common autoimmune disease with an incidence of roughly 3.9 cases per 100,000 person-years [7]. While the etiology of primary ITP is currently unknown, causes of secondary ITP include other autoimmune disorders, infections, vaccinations, lymphoproliferative disorders, congenital immune deficiencies, and medications. Diagnosis of ITP requires a platelet count of less than $100,000 / \mathrm{mm}^{3}$ in the absence of splenomegaly with no evidence of an etiology for a secondary thrombocytopenia [8].

The incidence of ITP in patients with malignancies is unclear. The largest reported case series consists of ten patients with concurrent ITP and breast cancer [9]. Its mechanism in these patients is unknown but may be related to tumor infiltration of bone marrow, chemotherapy-induced or radiation-induced bone marrow hypoplasia, or platelet consumption due to disseminated intravascular coagulation [9]. Most of these patients with breast cancer who developed ITP demonstrated hormone receptor positivity with advanced progression of their cancers, specifically with bony metastases [10].

Standard first-line therapy for ITP is a 2- to 4-week course of corticosteroids dosed at $0.5-2 \mathrm{mg} / \mathrm{kg}$, followed by a slow steroid taper [8]. Intravenous immunoglobulins or anti-RhD are sometimes required in conjunction with steroid therapy to stop acute bleeding. $80-90 \%$ of patients have a partial or complete response to steroid therapy [11]. Therapeutic options for refractory ITP include a splenectomy, as well as biologic agents such as rituximab, romiplostim, and eltrombopag [8], with $70-80 \%$ of patients achieving at least partial response following splenectomy [12].

\section{Conclusion}

We report the case of a patient whose ITP acutely presented twenty-four hours after a mastectomy and lymph node dissection for invasive ductal carcinoma of the breast. Her thrombocytopenia and acute bleeding responded well to standard corticosteroid therapy and conservative measures. Based on this case and other reported cases in breast cancer patients, ITP should be considered in the differential diagnosis for postoperative bleeding in patients undergoing mastectomy and lymph node dissection.

\section{Conflict of Interests}

The authors declare that there is no conflict of interests regarding the publication of this paper.

\section{References}

[1] R. Stasi and A. C. Newland, "ITP: a historical perspective," British Journal of Haematology, vol. 153, no. 4, pp. 437-450, 2011.

[2] K. Fink and H. Al Mondhiry, "Idiopathic thrombocytopenic purpura in lymphoma," Cancer, vol. 37, no. 4, pp. 1999-2004, 1976.

[3] R. Gencosmanoglu, P. Unalan, G. Kir, and R. Inceoglu, "Breast cancer and immune thrombocytopenic purpura: is there any association between these 2 distinct diseases?" Breast Care, vol. 2, no. 5, pp. 317-320, 2007.

[4] L. F. Porrata, S. Alberts, C. Hook, and C. A. Hanson, "Idiopathic thrombocytopenic purpura associated with breast cancer: a case report and review of the current literature," American Journal of Clinical Oncology, vol. 22, no. 4, pp. 411-413, 1999.

[5] C. Ustun, P. Dainer, and L. Hendricks, "Association of breast cancer and immune thrombocytopenic purpura," Southern Medical Journal, vol. 95, no. 11, pp. 1335-1337, 2002.

[6] O. W. Cummings and M. T. Mazur, "Breast carcinoma diffusely metastatic to the spleen: a report of two cases presenting as idiopathic thrombocytopenic purpura," American Journal of Clinical Pathology, vol. 97, no. 4, pp. 484-489, 1992.

[7] P. E. Abrahamson, S. A. Hall, M. Feudjo-Tepie, F. S. MitraniGold, and J. Logie, "The incidence of idiopathic thrombocytopenic purpura among adults: a population-based study and literature review," European Journal of Haematology, vol. 83, no. 2, pp. 83-89, 2009.

[8] S. Lakshmanan and A. Cuker, "Contemporary management of primary immune thrombocytopenia in adults," Journal of Thrombosis and Haemostasis, vol. 10, pp. 1988-1998, 2012.

[9] R. P. de Latour, G. des Guetz, V. Laurence et al., "Breast cancer associated with idiopathic thrombocytopenic purpura: a single center series of 10 cases," American Journal of Clinical Oncology, vol. 27, no. 4, pp. 333-336, 2004.

[10] M. A. Samimi, N. Mirkheshti, M. Heidarpour, and M. Abdollahi, "Idiopathic thrombocytopenic purpura in women with breast cancer," Journal of Research in Medical Sciences, vol. 15, no. 6, pp. 359-363, 2010.

[11] T. Sailer, K. Lechner, S. Panzer, P. A. Kyrle, and I. Pabinger, “The course of severe autoimmune thrombocytopenia in patients not undergoing splenectomy," Haematologica, vol. 91, no. 8, pp. 1041-1045, 2006.

[12] K. Kojouri, S. K. Vesely, D. R. Terrell, and J. N. George, "Splenectomy for adult patients with idiopathic thrombocytopenic purpura: a systematic review to assess long-term platelet count responses, prediction of response, and surgical complications," Blood, vol. 104, no. 9, pp. 2623-2634, 2004. 


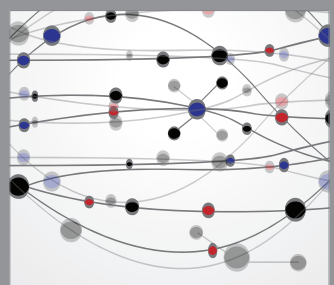

The Scientific World Journal
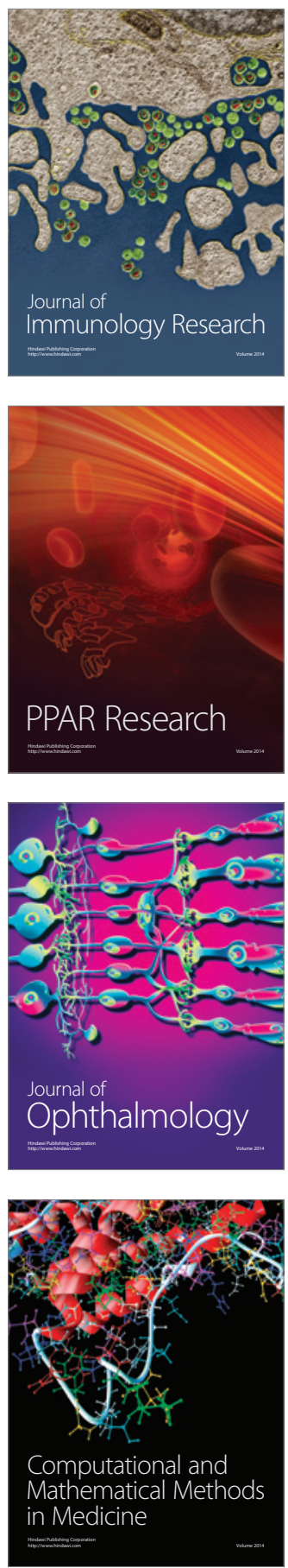

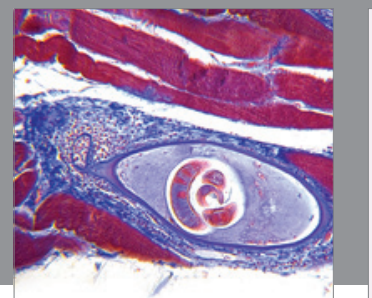

Gastroenterology

Research and Practice
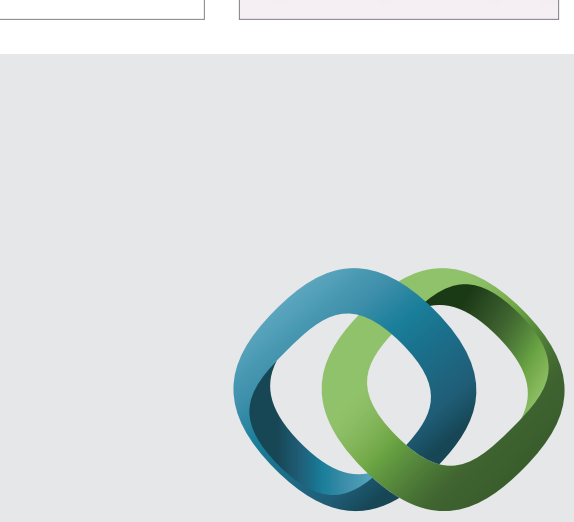

\section{Hindawi}

Submit your manuscripts at

http://www.hindawi.com
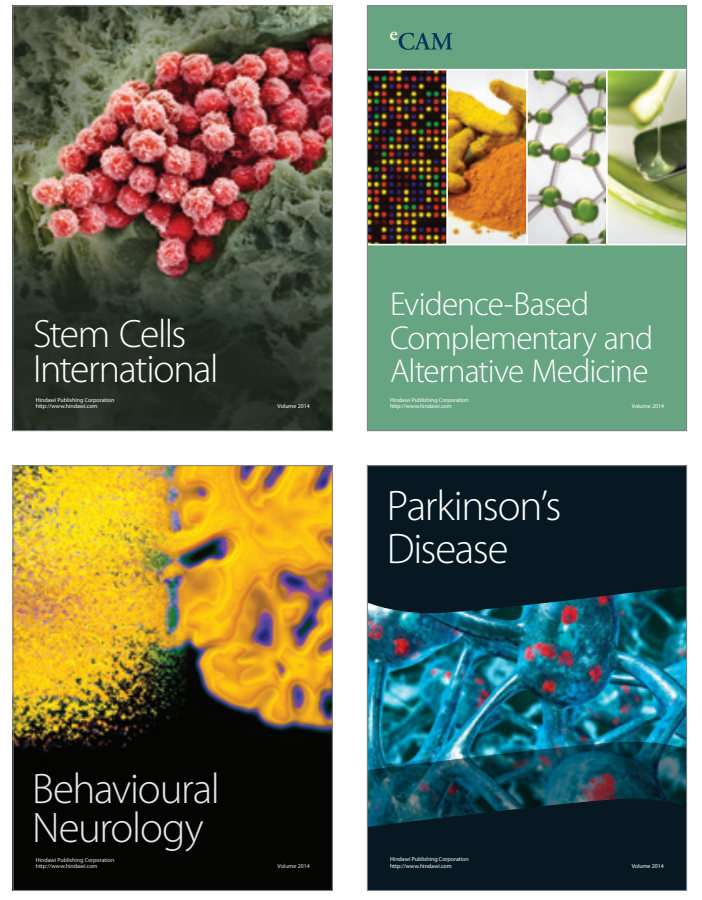
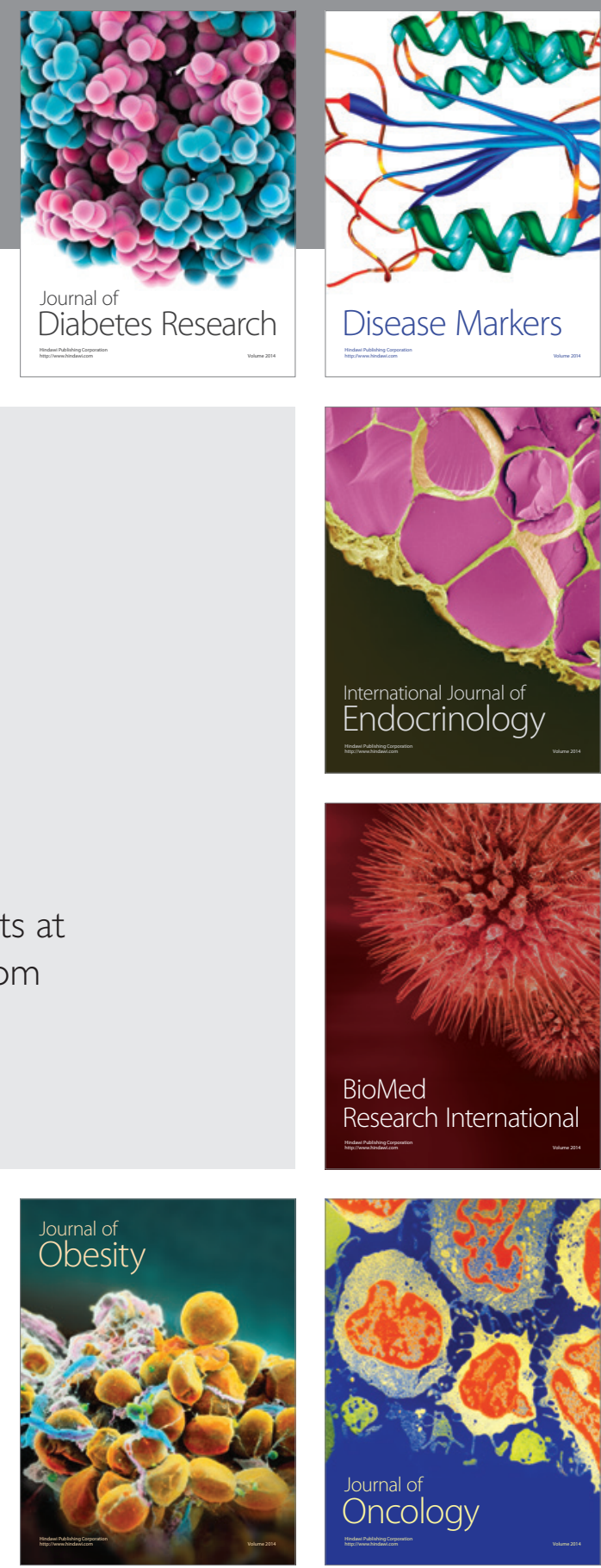

Disease Markers
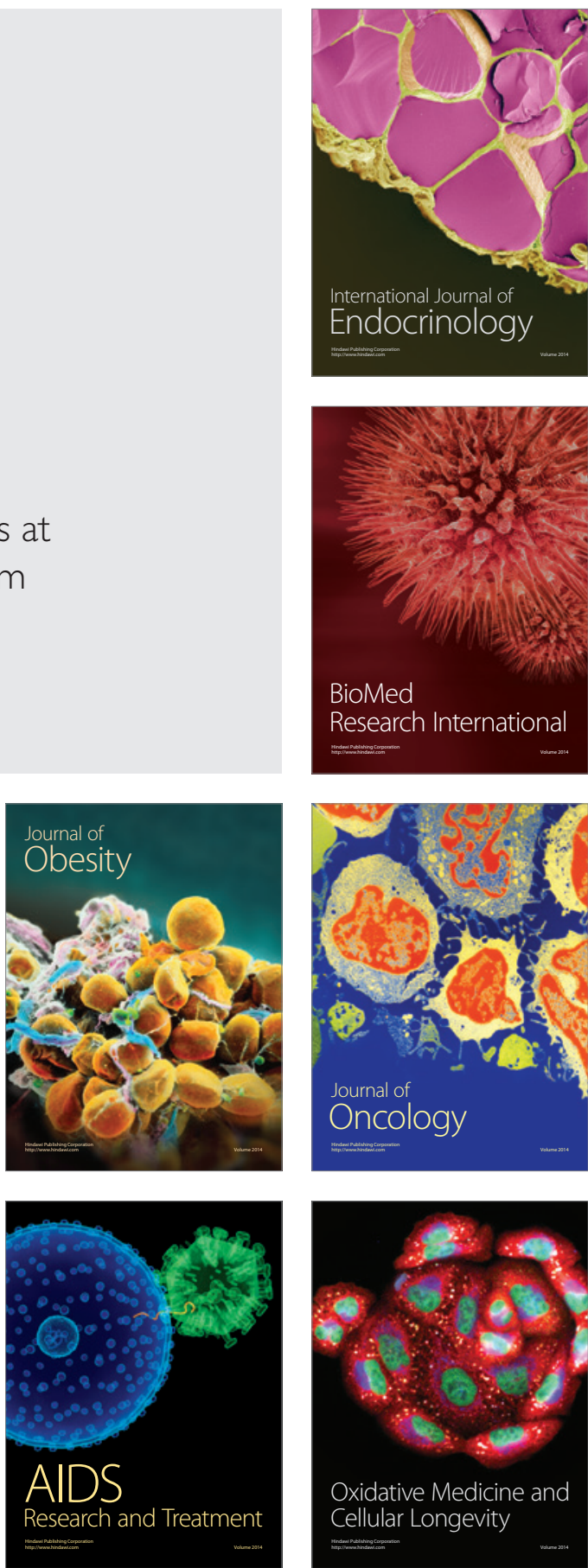